\title{
La dimensione dello spazio pubblico nelle conurbazioni di fondovalle. Ambiti e prospettive progettuali
}

\author{
Silvia Restelli \\ Dipartimento di Architettura e Studi Urbani, DASTU, Politecnico di Milano, Italia \\ silvia.restelli@polimi.it \\ Viviana di Martino \\ Dipartimento di Architettura e Studi Urbani, DASTU, Politecnico di Milano, Italia \\ viviana.dimartino@polimi.it
}

\begin{abstract}
Il contributo affronta il tema delle criticità riguardanti le conurbazioni lineari dei fondovalle alpini in relazione alla dimensione dello spazio pubblico. A partire dal riconoscimento degli impatti spaziali e relazionali causati dallo sviluppo della moderna urbanizzazione, il contributo individua possibili ambiti di progetto per radicare un nuovo sistema di spazi pubblici e collettivi capace di rispondere alla domanda congiunta di diritto alla città e alla natura espressa dagli abitanti del cosiddetto periurbano alpino. Gli spazi individuati, e portati ad esempio anche attraverso progetti, sono diffusi negli eterogenei paesaggi della montagna e costituiscono opportunità di intervento alle diverse quote per restituire qualità e identità ai territori di fondovalle e per ricomporre relazioni interrotte o incompiute attraverso il disegno di un nuovo sistema di spazi pubblici, collettivi e aperti che si sostanzia come vero e proprio progetto di rigenerazione del paesaggio fatto di luoghi, relazioni e identità.
\end{abstract}

\section{Keywords}

Paesaggio, spazio pubblico, fondovalle, città-montagna

\begin{abstract}
The paper deals with the critical issues concerning the public spaces and linear settlements 10 cated in the Alpine valley floors. The development of modern urbanisation caused spatial and relational impacts increasing the demand for the right to the city and nature expressed by the inhabitants of the Alpine suburbs. Therefore, the paper identifies possible project areas for establishing a new system of public and collective spaces, as already tested through the mentioned case studies concerning the different contexts. These selected spaces are widespread in heterogeneous mountain landscapes and constitute design opportunities at different elevations to restore territorial quality and identity and reconnect the interrupted or incomplete relationships. They could compose a new public, collective, and open spaces system to be developed as a real landscape regeneration project made of places, relationships, and identities.
\end{abstract}

\section{Keywords}

Landscape, public space, valley floor, city-mountain 


\section{Conurbazioni di fondovalle e spazio pubblico: una questione aperta ${ }^{1}$}

La riflessione proposta guarda a quei fondovalle alpini investiti nel tempo da consistenti fenomeni di urbanizzazione che ne hanno modificato i caratteri paesaggistici, consumando suolo, eliminando e frammentando gli spazi aperti, producendo conurbazioni lineari spesso di scarsa qualità costituite da un susseguirsi di spazi costruiti e infrastrutture che si configurano come cesure fisiche e percettive (Lassini, 2014). Lo sviluppo di questi contesti si lega a diversi fattori, tra cui quelli economici, socioculturali, morfologici, nonché alla prossimità con le aree metropolitane pedemontane. In particolare, la morfologia altimetricamente livellata e l'accessibilità favorevole hanno contribuito alla diffusione degli spazi della città di matrice fordista (Dematteis, 2016) e post-fordista (Perlik, 2019) concorrendo alla formazione del cosiddetto periurbano alpino (Corrado, 2016) che occupa diverse valli, sviluppandosi in relazione alle specificità dei contesti.

Come già proposto da Lanzani e Curci (2018), alcuni fondovalle possono essere descritti come contesti 'intristiti' in quanto le urbanizzazioni che li occupano faticano a 'farsi città' a causa dell'omologazione spaziale e dell'assenza di luoghi e occasioni di relazione, sebbene in passato le città costituissero l'armatura del territorio montano fondata su un rapporto stretto con gli ambienti rurali e naturali (Dematteis, 1975). Il bisogno e il diritto alla città
(Lefebvre, 1974) costituiscono istanze di forte attualità per quei contesti montani laddove il processo di urbanizzazione ha prodotto prevalentemente agglomerati edilizi, tralasciando la dimensione immateriale della città quale civitas, ovvero quell'insieme fondamentale di relazioni sociali, usi, servizi, luoghi per la collettività (Dematteis, 2012). In questo senso è crescente la necessità di sperimentare modelli insediativi capaci di coniugare i valori urbani esistenti e potenziali con le risorse e i valori degli ambienti naturali e rurali propri dei contesti montani.

Il portato delle conurbazioni di fondovalle ha caratteri tangibili e intangibili. I primi sono connessi a quei processi di espansione degli insediamenti che, laddove guidati da una prevalente logica privatistica, hanno prodotto una giustapposizione di elementi ed usi spesso privi di un disegno di insieme a discapito dello spazio pubblico inteso come spazio per la collettività. Invece, i portati immateriali si configurano come relazioni interrotte o rapporti mancati tra le aree urbane e il loro intorno, ma anche come uno scollamento tra comunità locali e territorio, tra individui e comunità. Pertanto, i processi di sviluppo non territorializzato possono avere conseguenze non solo fisiche ma anche implicazioni morali e sociali connesse alla perdita di spazi di relazione. La necessità di ricucire, rigenerare e risignificare i territori del fondovalle pas- 


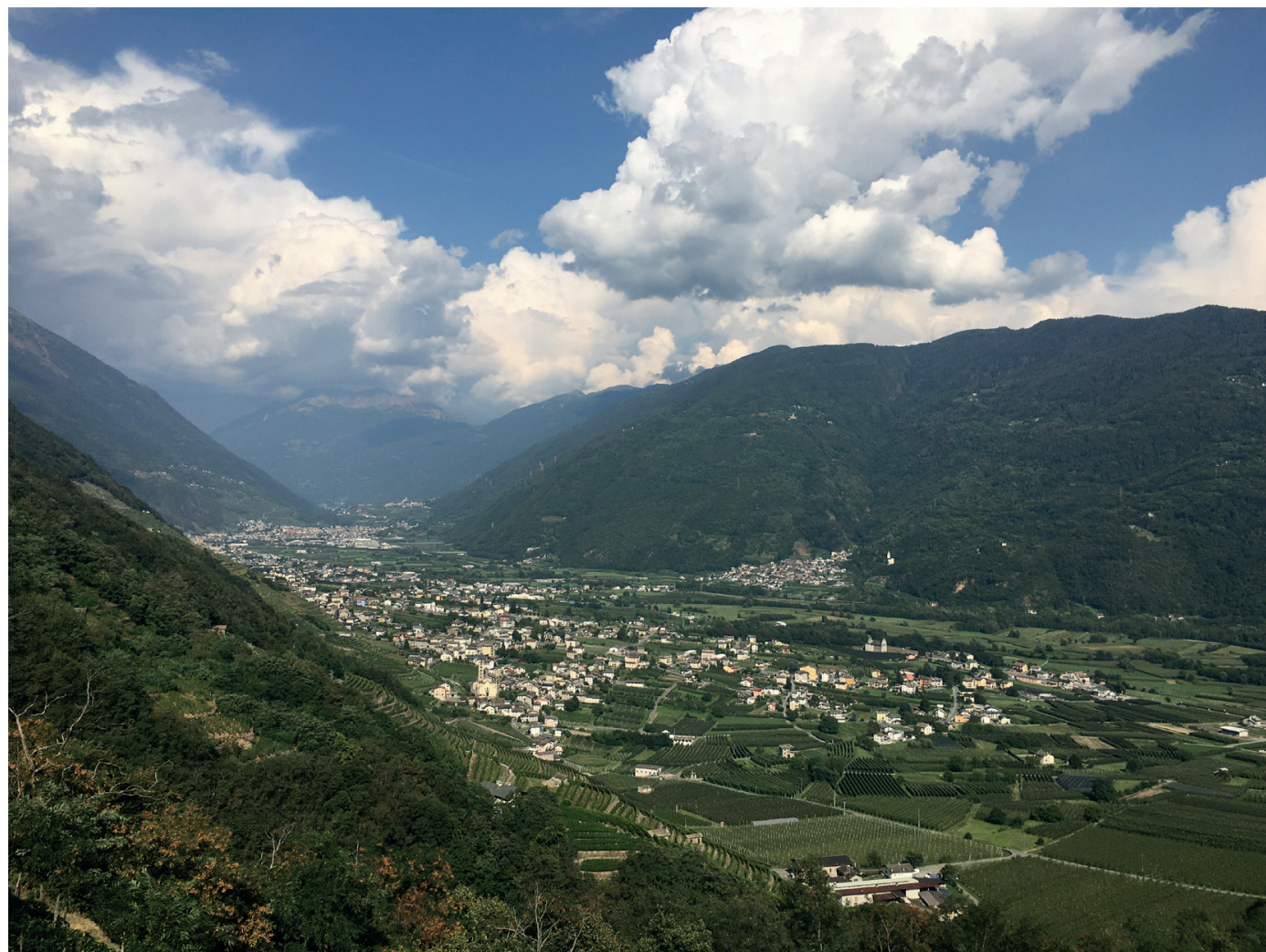

Fig. 1 - Il fondovalle della Valtellina da Teglio a Tirano (SO) (Foto di: Silvia Restelli, 2020).

sa quindi attraverso istanze collettive dal carattere fisico e immateriale.

È in questo quadro che il sistema degli spazi aperti assume potenzialmente un ruolo sociale e spaziale consistente, facendosi portatore di un valore di urbanità che, in un disegno di insieme di luoghi e connessioni, da un lato possa farsi carico delle diverse istanze a cui lo spazio pubblico è chiamato a rispondere quale elemento chiave per il benessere individuale e collettivo (INU, 2013), dall'altro costituisca l'occasione per rinnovare la concezione stessa di spazio pubblico entro una dimensione paesaggistica (Mattiucci, 2018a) che è parte integrante dell'identità di tali contesti.

La riflessione proposta muove da una interpretazione dello spazio collettivo in chiave estensiva, quale bene comune che prescinde dalla sua natura 'pub- sti di diversa natura in una dimensione più ampia del progetto, volta a promuovere una riappropriazione fisica e sociale dei luoghi da parte di chi li vive, ma anche ad attivare processi diffusi di rigenerazione, riqualificazione, rivitalizzazione, cura e presidio territoriale improntati alla sostenibilità. Proprio in questi contesti, dove logiche di concentrazione e sovrautilizzo hanno determinato uno sviluppo insediativo talvolta privo di urbanità, si sono affiancate dinamiche opposte di contrazione e abbandono (Restelli, 2021) che hanno generato una capillare presenza di aree di scarto, spazi aperti dimenticati o sottoutilizzati, che possono rappresentare ambiti strategici nei quali attivare progettualità per restituire qualità e identità ai territori di fondovalle e per ricomporre alle diverse scale quel sistema di relazioni interrotte o incompiute. 

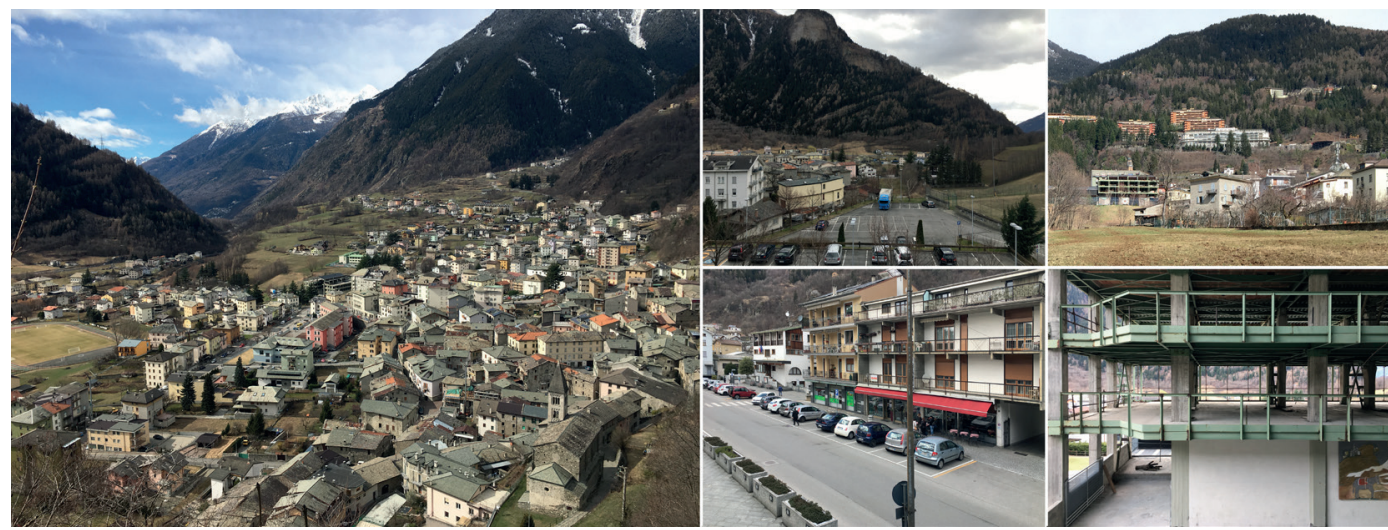

Fig. 2 - Il centro urbano di Sondalo (SO) (Foto di: Silvia Restelli, 2019).

Questi spazi possono riconfigurarsi come opportunità per innestare un nuovo sistema di spazi pubblici e collettivi che unisca gli ambiti sconnessi e frammentati del fondovalle con il sistema degli spazi aperti alle diverse quote in un disegno complessivo di luoghi e relazioni che si sostanzia come vero e proprio progetto di paesaggio.

\section{Paesaggio urbano: rigenerazione e vivibilità}

In uno scenario come quello descritto, sono innanzitutto i contesti propriamente urbani quelli in cui il 'diritto alla città', nelle sue molteplici implicazioni, si può realizzare attraverso un progetto di spazio pubblico che diviene esso stesso progetto di paesaggio, restituendo valori tangibili e intangibili a quei contesti ordinari che costituiscono l'ambiente di vita quotidiano delle persone (CoE, 2000).

II progetto si rivolge quindi ad un paesaggio urbano che possa ricostituirsi in prima istanza attraverso interventi mirati di rigenerazione che abbiano per oggetto le centralità storiche su cui si fonda, da recuperare nel rispetto dei caratteri originari e simbolici dei luoghi e con l'obiettivo più ampio di riattivare anche quel tessuto capillare di attività e funzioni che contribuiscono a rendere lo spazio pubblico vitale e attrattivo (di Martino, 2021).

Un paesaggio urbano che nella sua repentina crescita ha occupato spazi senza riuscire a costruire luoghi e a cui è possibile restituire qualità e identità rigenerando quei comparti dismessi che costituiscono uno dei lasciti delle dinamiche di contrazione che lo hanno progressivamente investito (Arcidiacono et al., 2021) e che possono rappresentare nuove occasioni per ricreare urbanità laddove manca, coinvolgendo nel progetto anche quei servizi e quelle attrezzature collettive che costituiscono un baluardo di città pubblica spesso scarsamente valorizzato nelle sue potenzialità di aggregazione.

Un paesaggio urbano, infine, in cui il diritto alla città può essere letto anche come diritto alla natura (Mattiucci, 2018b), laddove alla originaria mancanza di spazi verdi propria degli insediamenti storici, un tempo compensata dalla prossimità con lo spazio aperto e facilmente fruibile del fondovalle, lo sviluppo insediativo moderno ha risposto promuovendo un modello abitativo frammentato e introverso in cui lo spazio aperto diviene lo spazio del giardino privato.

Paradossalmente, mentre nelle città del XX secolo cresceva la consapevolezza dei benefici connessi alla presenza di parchi e aree verdi quali luoghi di decompressione e aggregazione in risposta al nuovo modello di vita urbano (Fein, 1972), il cosiddetto periurbano alpino prendeva forma spesso proprio quale meta di soggiorno turistico o terapeutico a beneficio dei cittadini di pianura, da un lato contribuendo ad erodere la consistenza del capitale naturale e 


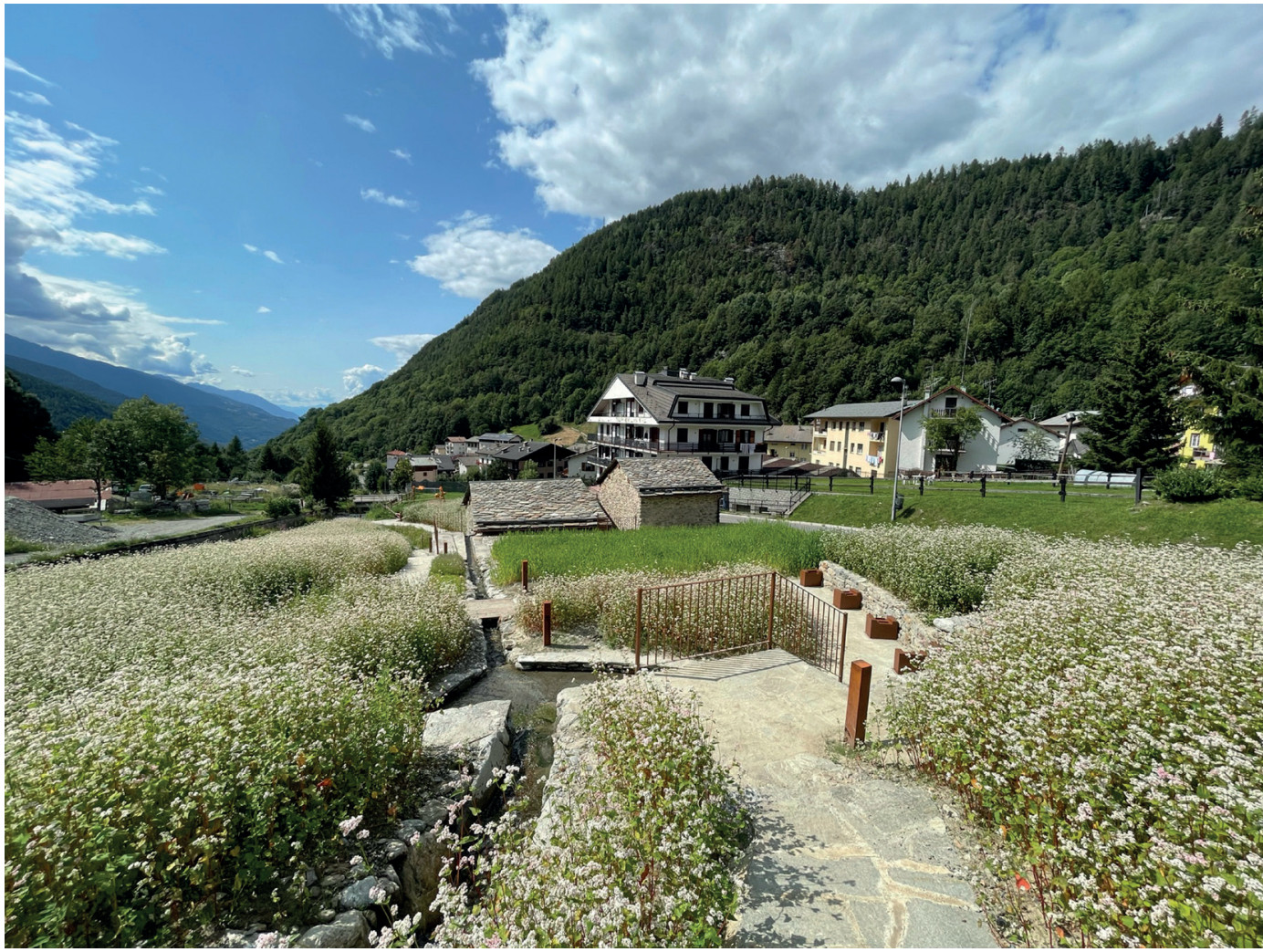

Fig. 3 - Mulino dei Plaz di Aprica (SO) (Foto di: Silvia Restelli, 2021).

paesaggistico che ne costituiva la primaria attrattiva e dall'altro trascurando la realizzazione di luoghi e spazi di qualità per chi lo abita.

II diritto alla natura è invocato oggi attraverso i principi che guidano lo sviluppo urbano sostenibile (UN, 2015, SDG 11) e può essere esercitato in questi contesti sfruttando, ad esempio, la disponibilità di aree agricole intercluse e di spazi aperti interstiziali che, depotenziati nel loro carattere produttivo di matrice storica, potrebbero divenire centrali in un disegno di insieme che sappia rileggerli in chiave fruitiva.

Emblematico in tal senso è il caso di Sondalo, piccolo comune valtellinese che lega il suo sviluppo moderno alla realizzazione di un imponente sanatorio, oggi in parte dismesso o riconvertito, e che racchiude in sé un'ampia casistica di opportunità pro- se, anche se oggetto di svariate proposte (Del Curto, Menini, 2018). Se da un lato, alcuni interventi recenti hanno valorizzato il principale asse urbano della città moderna, restituendo qualità allo spazio pubblico su cui si affaccia buona parte delle attività commerciali del comune, svariati nodi restano invece ancora irrisolti. Tra questi emergono gli spazi aperti del centro storico, ricchi di valore identitario ma svuotati di usi e funzioni, così come gli ambiti agricoli di fondovalle, che costituiscono uno scenario paesaggistico significativo accerchiato da una sequenza di recinti che delimitano edifici residenziali e attrezzature senza costruire un rapporto con il contesto. Su quello stesso asse si staglia la sagoma fuori scala del centro parrocchiale, un'ambizione urbana sovradimensionata solo in parte completata, Iasciando in una posizione nodale un ingombrante schele- 


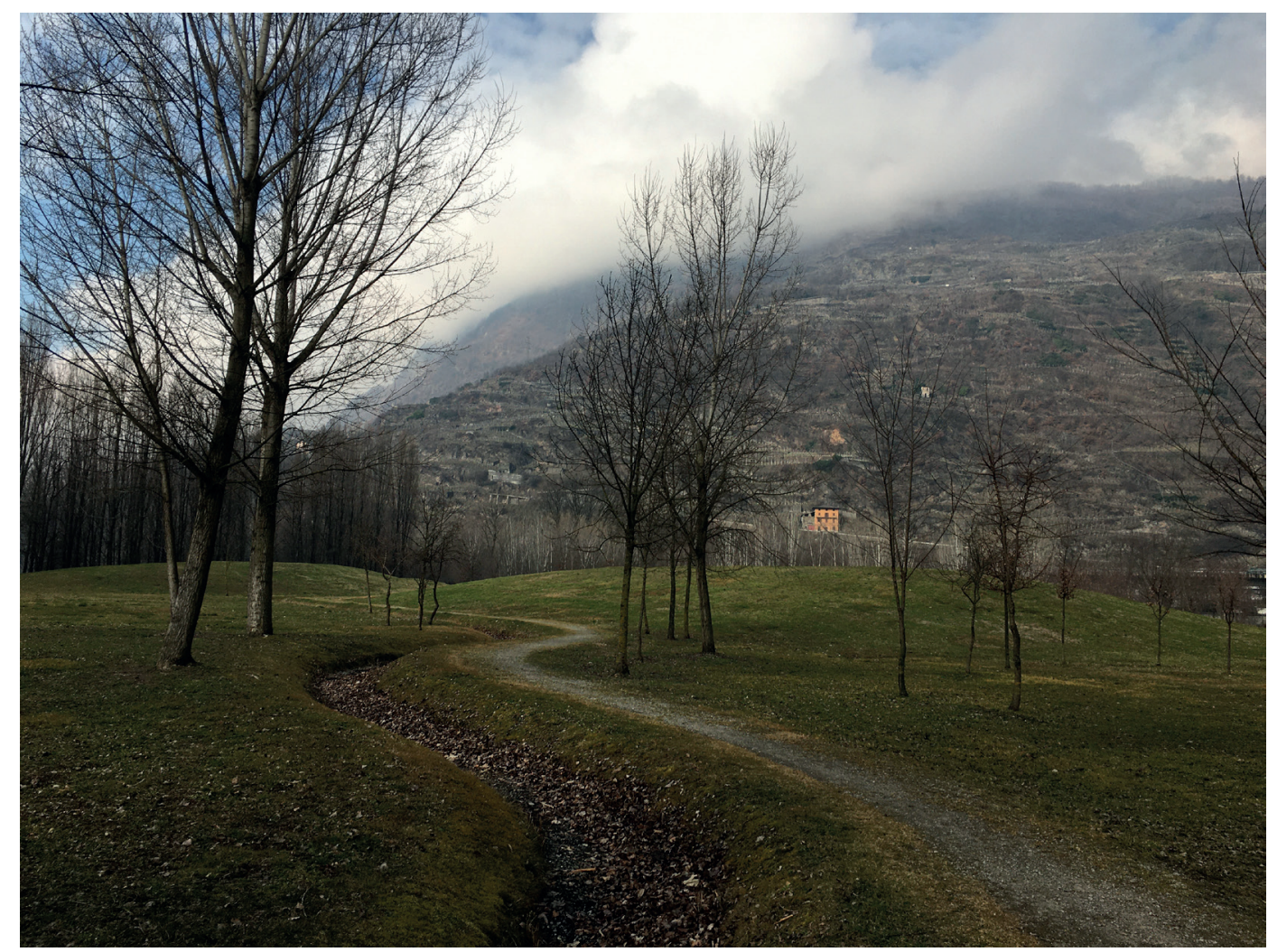

Fig. 4 - Parco Adda-Mallero, Sondrio (Foto di: Silvia Restelli, 2020).

tro che potrebbe costituire una preziosa opportunità di rigenerazione per costruire nuove relazioni spaziali e percettive.

La rigenerazione di aree dismesse o sottoutilizzate può assumere un ruolo rilevante se guidata da processi che mettano a sistema i valori propri dei luoghi oggetto di trasformazione intercettando i diversi attori, i bisogni della comunità nonché qualificando il paesaggio. In questo senso, il caso del Mulino dei Plaz ad Aprica costituisce un esempio di spazio restituito alla comunità attraverso un progetto integrato di recupero dei beni costruiti e degli spazi aperti, valorizzando il carattere storico identitario del luogo e rendendolo fruibile per lo svolgimento di pratiche sociali.

\section{Paesaggio fluviale: sostenibilità e rivitalizzazione}

Nei fondovalle fortemente inurbati, accanto agli spazi aperti residuali appena descritti, alcuni preziosi elementi di naturalità permangono, pur trovandosi spesso in contesti posti al margine o in condizione di compressione e frammentazione. Tra questi, il principale elemento di naturalità, in essere o potenziale, è rappresentato dal fiume che, oltre a strutturare longitudinalmente le valli, costituisce un bene comune sul quale attivare progettualità sostenibili per la valorizzazione del capitale naturale che possano costituire anche occasione per realizzare interventi di carattere fruitivo in grado di riavvicinare la comunità al suo fiume.

La riattivazione dei corsi d'acqua e degli spazi perifluviali costituisce un'ulteriore opportunità progettuale per rispondere alla diffusa domanda sociale di 


\section{qualificazione del capitale naturale.}

Strategie di riattivazione e rinaturalizzazione fluviale guidate da principi di sostenibilità sono già al centro di alcune politiche di sistema in diversi settori della regione alpina, al fine di sostenere misure e progetti per migliorare la qualità del fiume e degli ambiti perifluviali restituendo loro centralità nel fondovalle quali contesti qualificati e qualificanti. In Svizzera, ad esempio, una politica avviata nel 2011 sostiene interventi che agiscono su morfologia e funzionalità dei corsi d'acqua attraverso azioni che coinvolgono habitat naturali, alvei e argini con progettualità dal carattere fortemente multifunzionale che integrano naturalità, ecologia, sicurezza, fruizione, turismo e questioni sociali (Castelli, 2014). Similmente, la Provincia Autonoma di Bolzano definisce, gestisce e coordina misure per la rinaturalizzazione attraverso uno strumento di pianificazione che si sostanzia mediante azioni specifiche di rigenerazione del capitale naturale e diminuzione dei fattori di rischio idrogeologico, incrementando al contempo gli accessi al fiume e la dotazione di spazi ricreativi (Blaas, Hecher, 2018). Un esempio di integrazione degli spazi aperti del paesaggio fluviale in un disegno di sistema che lavora a diverse scale è ritrovabile nel fondovalle valtelline-
Un progetto di scala territoriale è rappresentato dalla ciclabile che accompagna il fiume ricucendo ambiti naturali, rurali e urbani e diventando un'occasione fruitiva per abitanti e turisti. II valore del fiume come ossatura portante del capitale naturale di fondovalle è stato sostenuto dal progetto Grande Foresta di Fondovalle valtellinese che ha potenziato la dotazione di spazi boschivi in prossimità del fiume ricostruendo parte del patrimonio ambientale cancellato (Lassini, 2014). Guardando invece alla scala locale, il parco Adda-Mallero di Sondrio costituisce un nuovo luogo pubblico dal carattere più urbano che offre alla comunità locale spazi per attività ricreative a stretto contatto con elementi naturali. Il parco non solo restituisce ai cittadini uno spazio precedentemente inaccessibile e degradato, ma con interventi mirati rivitalizza e potenzia la qualità degli ambienti fluviali e perifluviali.

\section{Paesaggio periurbano: presidio e riconoscibilità}

Nel paesaggio periurbano la dimensione dello spazio aperto acquisisce maggiore consistenza assumendo i caratteri delle aree agricole di fondovalle, ma è nel rapporto irrisolto tra usi e funzioni degli spazi aperti di margine che può prendere forma un nuovo progetto di ricucitura e risignificazione a cui associare anche un valore di presidio sociale, oltre che ambientale e territoriale.

Le possibilità di progetto risiedono negli ambiti frammentati o parzialmente erosi dall'urbanizzazione, in cui le pratiche agricole permangono negli usi ma risultano banalizzate nelle forme e nei caratteri e in cui spesso si è rinunciato a costruire una relazione compiuta con l'urbano. II tema su cui lavorare per dare nuova identità a questi contesti è in via prioritaria quello della multifunzionalità, ripristinando il valore sociale, ecologico e paesaggistico associato alle pratiche agricole (Maier, Shobayashi, 2001) al fine di promuovere una visione integrata tra spazio edificato e spazio aperto basata su relazioni virtuose e non univoche (Davoudi, Stead, 2002). 

rale, come sperimentato in alcune interessanti iniziative di new farming (Gretter et al., 2019). È in questo quadro che si inseriscono alcune recenti esperienze sviluppate nell'ambito di progetti finanziati dal programma INTERREG Alpine Space che vedono coinvolte diverse realtà italiane ed europee.

II progetto LOS_DAMA! Landscape and Open Space Development in Alpine Metropolitan Area ha come obiettivo la gestione e la valorizzazione dei contesti di margine del periurbano alpino per costruire una rete di spazi fruibili che contribuiscano ad incrementare la vivibilità dei sistemi urbani e a riavvicinare le persone ad un capitale naturale e culturale spesso poco sfruttato.

Tra le azioni sviluppate per le aree pilota risulta di grande interesse il caso di Trento che ha lavorato sul potenziamento delle relazioni tra urbano e periurbano dedicando particolare attenzione, da un lato, alla ricognizione delle aree agricole di margine sottoutilizzate, dall'altro, al coinvolgimento della cittadinanza a diversi livelli, con l'obiettivo di costruire reti non solo fisiche ma anche sociali per una valorizzazione multifunzionale di tali spazi.

Anche il progetto LUIGI Linking Urban and Inner-AIpine Green Infrastructure - Multifunctional Ecosystem Services for more liveable territories ${ }^{3}$ è incen- reti verdi periurbane in ambito alpino ma con un approccio di scala più ampia, con l'obiettivo di mantenere e implementare connessioni ecologiche ma anche economiche e sociali durevoli tra i contesti rurali e urbani dello Spazio Alpino.

\section{Paesaggio di versante: cura e fruizione}

Infine, in un ragionamento che ponga al centro gli specifici caratteri del contesto alpino, anche il paesaggio di versante diviene parte attiva nella costruzione dello spazio collettivo in senso esteso, richiamando idealmente il ruolo che storicamente i commons hanno avuto per le comunità montane (Mocarelli, 2013). Esso costituisce un paesaggio contraddistinto da dinamiche di abbandono e contrazione che si riflettono nella mancata gestione, se non addirittura nella perdita, di un capitale naturale e culturale di inestimabile valore che proprio nella ricostruzione del rapporto con il fondovalle può trovare nuove occasioni di valorizzazione. Così, in una concezione che guarda al paesaggio come spazio pubblico e collettivo, è possibile interpretare i contesti di versante come ambiti di azione per la ricucitura tra ambiente urbano e spazi aperti alle diverse quote.

II paesaggio di versante si costituisce come un ambito variegato dove biodiversità ed etnodiversità si articolano in un mosaico eterogeneo. Sebbene gli spazi aperti presentino un grande valore naturale, essi costituiscono anche un rilevante valore culturale in quanto il paesaggio che determinano "è formato da stratificazioni della memoria almeno quanto da sedimentazioni di rocce" (Shama, 1997, p.7).

Molti degli spazi aperti di versante sono storicamente contraddistinti da una forte dimensione collettiva testimoniata dal diritto di uso civico esercitato dalla comunità sul suo territorio (Pallottino, 2014). I benefici diretti e indiretti di tali usi, legati ad esempio al pascolo o alla selvicoltura, si fondano su un principio di solidarietà a vantaggio del singolo e dell'intera comunità locale. A questo sistema di spazi aperti si sovrappone una rete di sentieri che 


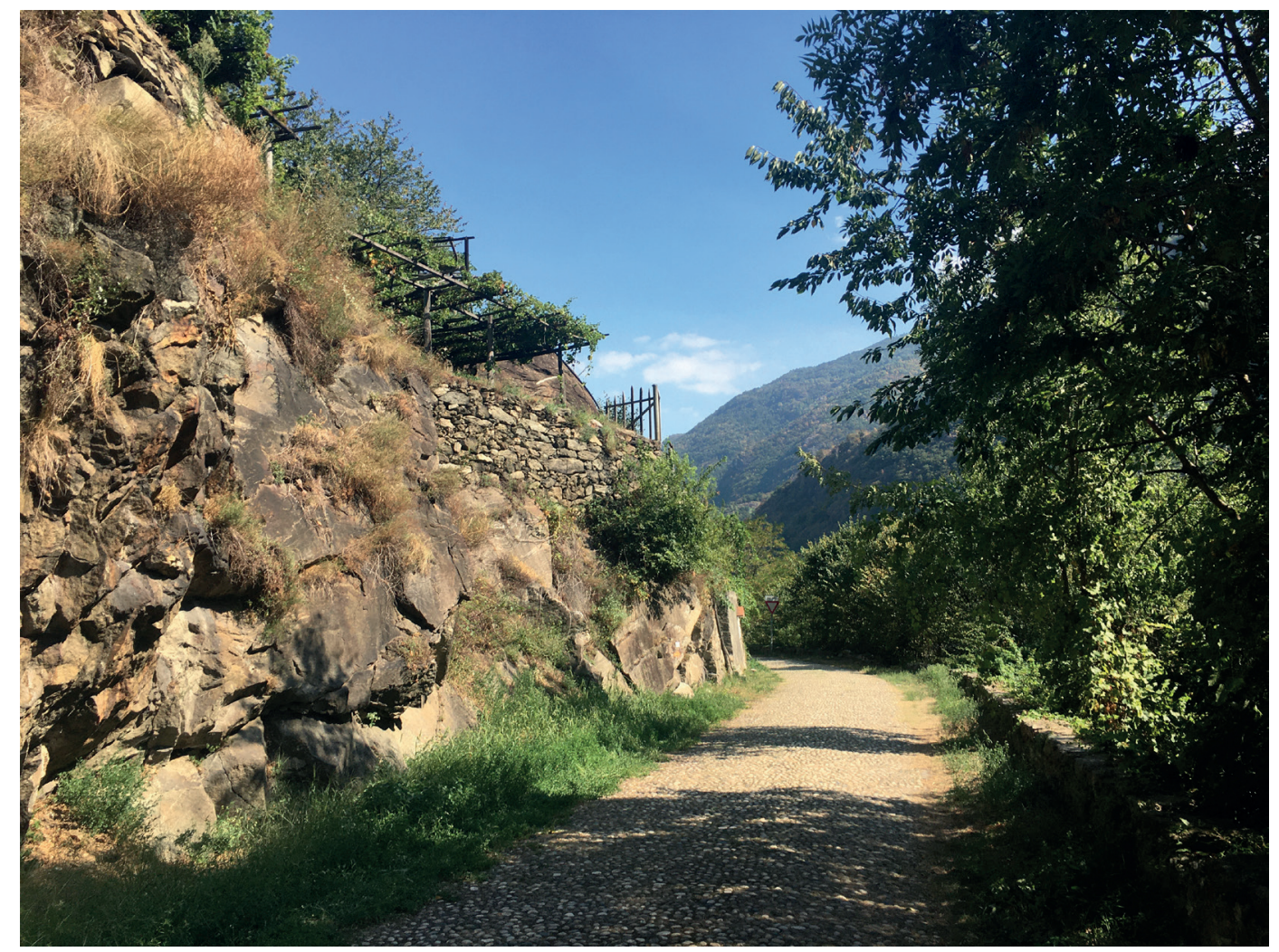

Fig. 6 - Via Francigena tra Verrès e Pont-Saint-Martin (AO) (Foto di: Viviana di Martino, 2020).

innervano capillarmente i rilievi, usati dalla comunità locale come dai viandanti per spostarsi di valle in valle. Strade, sentieri e mulattiere hanno contribuito così a rendere fruibili e vivibili le valli concorrendo alla definizione del concetto di Heimat (Salsa, 2019). La rete della viabilità storica, e il cospicuo patrimonio costruito minuto e diffuso che la accompagna, costituiscono testimonianze preziose della storia culturale, sociale ed economica delle comunità che li hanno prodotti imprimendo linearmente sul territorio il segno del loro passaggio. Lungo tale rete si ritrovano non solo insediamenti stabili o temporanei e spazi agricoli, ma anche fontane per l'abbeveramento, simboli religiosi ed altri elementi puntuali collocati a supporto di coloro che percorrevano i sentieri quotidianamente o per spostamenti di lungo raggio.
Anche oggi i percorsi che innervano i versanti possono essere valorizzati come spazi fruitivi pubblici e collettivi dal carattere quotidiano, così come elementi connettivi alla scala vasta per ricucire molteplici contesti.

Il caso dell'area metropolitana di Grenoble, ad esempio, propone un approccio strategico volto ad integrare nella quotidianità degli abitanti della conurbazione l'uso e la fruizione dei paesaggi di versante potenziando e valorizzando il sistema di connessioni trasversali all'andamento dell'area urbana di valle (Ambrosino, Buyck, 2018). La ricucitura tra i due contesti sostiene la stretta interrelazione tra spazi pubblici e collettivi di fondovalle e spazi aperti di versante, sottolineando il valore collettivo di questi ultimi. La presenza di una rete fruitiva si costituisce come uno spazio collettivo che lega il siste- 
ma urbano e il suo intorno, interpretando le aree alle quote più elevate come un giardino urbano di scala sovralocale (Mattiucci, De Marco, 2013).

L'organizzazione in percorsi di lungo raggio può invece dare spazio ad esperienze fruitive dal carattere più immersivo prevedendo un tempo di percorrenza dilatato. Questi percorsi costituiscono un'occasione per valorizzare itinerari storico-culturali, come la via Francigena, o sentieri articolati lungo paesaggi tradizionali di versante come la Via dei Terrazzamenti retici della Valtellina.

Azioni di ripristino, manutenzione e messa a sistema della rete fruitiva, ma anche azioni puntuali di recupero del patrimonio costruito collocato lungo i percorsi, rappresentano misure per sostenere la costruzione di un'ampia rete di spazi ad uso pubblico e collettivo volta a ricucire connessioni fisiche ed immateriali interrotte, ma costituiscono anche misure di cura e manutenzione territoriale. Infatti, questa antica rete di connessioni, grazie alla sua estensiva e diffusa presenza anche nelle aree più marginali, si presenta come una potenziale rete di accessibilità a supporto di misure di gestione forestale, regolazione delle acque, controllo degli incendi e altre attività di governo del territorio (Cazzani, 2021).

\section{Le radici del progetto}

I temi e le strategie proposte ragionano sul progetto di spazio pubblico in senso esteso, interpretandolo come telaio per costruire sistemi di relazioni a geometria variabile che, lavorando con diversi materiali, consentano di coniugare la ricerca di migliori condizioni di vivibilità e qualità alla scala urbana, con una dimensione progettuale e strategica che si confronta con la scala del paesaggio e con i valori e le fragilità che lo caratterizzano. II rafforzamento delle relazioni tra le diverse scale di intervento diviene dunque la chiave tramite cui attivare processi di valorizzazione e cura delle risorse paesaggistiche e territoriali montane e di rivitalizzazione e rigenerazione dei contesti urbani di fondovalle in un continuo rapporto di reciprocità che coinvolga non solo la dimensione fisica del progetto ma anche sociale, identitaria, culturale e, non da ultimo, economica. In questo senso, la reinterpretazione in chiave multifunzionale dei luoghi di scarto, che nei contesti in esame si configurano come spazi aperti dimenticati o sottoutilizzati, diviene un'occasione per incidere sul senso di appartenenza delle comunità, anche per scongiurarne il rischio di sradicamento (Weil, 2002, p. 45) che rappresenta una delle cause alla base dei processi di abbandono, mancanza di cura e presidio territoriale.

La multifunzionalità del progetto di paesaggio costituisce inoltre un elemento di possibilità per la ri- 
generazione dei contesti montani, anche rispetto all'opportunità di coniugare la dimensione pubblica e privata del progetto mediante l'attivazione di una rinnovata partnership tra attori, il cui esito possa portare benefici materiali e immateriali al singolo come alla collettività.

L'attuazione delle strategie e delle progettualità proposte nel testo, infine, rimanda ad una vasta gamma di strumenti programmatici e di finanziamento che includono, tra gli altri, piani urbanistici alle diverse scale, protocolli come i contratti di fiume, programmi europei come Interreg Alpine Space, finanziamenti legati a politiche nazionali e regionali o a fondazioni come Cariplo e il FAI, così come l'attivazione di accordi di partenariato alla scala locale.

\section{Note}

${ }^{1}$ Il testo è esito condiviso del lavoro e della riflessione delle autrici. Sono comunque attribuibili a Silvia Restelli i paragrafi: Conurbazioni di fondovalle e spazio pubblico: una questione aperta; Paesaggio fluviale: sostenibilità e rivitalizzazione; Paesaggio di versante: cura e fruizione, e a Viviana di Martino i paragrafi: Paesaggio urbano: rigenerazione e vivibilità; Paesaggio periurbano: presidio e riconoscibilità; Le radici del progetto.

${ }^{2}$ Per approfondimenti si rimanda al sito: www.alpine-space.eu/projects/los_dama/en/home

${ }^{3}$ Per approfondimenti si rimanda al sito: www. alpine-space.eu/projects/luigi/en/home 


\section{Bibliografia}

Ambrosino C., Buyck J. 2018, The Mountain Metropolis's Land Design Project. Grenoble, from Plain to Slope, "Journal of Alpine Research | Revue de géographie alpine», n.106, vol.2.

Arcidiacono A., Pogliani L., Restelli S. 2021, Caratteri, dimensioni e tipologie della dismissione e dell'abbandono nei paesaggi lombardi. Prospettive di rigenerazione, in Arcidiacono A., Manfredi C. (a cura di), Ricerche e fotografia di paesaggio in Lombardia. Indagini sulle fragilità territoriali, Silvana Editoriale, Milano, pp. 58-79.

Blaas K., Hecher P. 2018, Revitalisierung der Fließgewässer Südtirols Hintergründe und Praxisbeispiele, Riqualificazione fluviale in Alto Adige Contesto generale ed esempi pratici, Provincia Autonoma di Bolzano - Alto Adige, Agenzia per la Protezione civile - Centro funzionale provinciale, Bolzano. Castelli S. 2014, Rivitalizzazione dei corsi d'acqua in Svizzera, in Bonardi L., Caligari A., Foppoli D., Gadola L., Grossi D., Stangoni T., Vanoi G. (a cura di), Paesaggi Valtellinesi. Trasformazione del territorio cultura e identità locale, Mimesis, Milano-Udine, pp. 237-243.

Cazzani A., Boriani M., 2021, The Role of Historic Roads to Preserve and Valorise the Landscape, in Pileri P., Moscarelli R. (a cura di), Cycling o Walking for Regional Development. How Slowness Regenerates Marginal Areas, Springer Nature Switzerland, pp.85-104.

CoE, Council of Europe, 2000, Convenzione Europea del Paesaggio, Firenze.

Corrado F. 2016, Abitare nei territori alpini di oggi: nuovi paradossi e l'esigenza di politiche abitative innovative, "Scienza del Territorio», n. 4, pp. 67-74.
Davoudi S., Stead D. 2002, Urban-Rural Relationships: an introduction and a brief history, «Built Environment», vol. 28, n. 4, pp. 269-277.

Del Curto D., Menini G. 2018, II consumo di suolo nella montagna lombarda. Un confronto tra piano e progetto urbano nel Novecento, in Arcidiacono A., Di Simine D., Ronchi S., Salata S., Consumo di suolo, servizi ecosistemici e green infrastructures: Caratteri territoriali, approcci disciplinari e progetti innovativi, INU Edizioni, Roma, pp. 83-91.

Dematteis G. 1975, Le Città Alpine, in Parisi B. (a cura di), Le Città Alpine. Documenti e note, Atti deI XXI Congresso Ceografico Italiano (Verbania 1971), Vita e Pensiero, Milano.

Dematteis G. 2012, La metro-montagna: una città al futuro, in Bonora P. (a cura di), Visioni politiche del territorio. Per una nuova alleanza tra urbano e rurale, Archetipolibri, Bologna, pp. 85-92.

Dematteis G. 2016, La città ha bisogno della montagna. La montagna ha diritto alla città, «Scienza del Territorio», n. 4, pp. 10-17.

Dematteis G. 2020, II manifesto di Camaldolie la centralità della montana, in Casanova L. (a cura di), Avere cura della montagna. L'Italia di salva dalla cima, Altraeconomia, Milano, pp. 23-36.

di Martino V. 2021, La valorizzazione della rete del commercio locale come dispositivo di rigenerazione urbana. Prospettive, strumenti e indirizzi operativi, in Caruso N., Pasqui G., Tedesco C., Vassallo I. (a cura di), Atti della XXIII Conferenza Nazionale SIU. DOWNSCALING, RIGHTSIZING. Contrazione demografica e Riorganizzazione spaziale, Politecnico di Torino, Planum Publisher, Roma-Milano, pp. 129-134. 
EC, European Commission 2013, Building a Green Infrastructure for Europe, Publications Office of the European Union, Bruxelles, Luxembourg.

Fein A. 1972, Frederick Law Olmsted and the American Environmental Tradition, George Braziller, New York.

Gretter A., Dalla Torre C., Maino F., Omizzolo A. 2019, New Farming as an Example of Social Innovation Responding to Challenges of Inner Mountain Areas of Italian Alps, «Journal of Alpine Research | Revue de géographie alpine», n.107, vol.2.

INU, Istituto Nazionale di Urbanistica, 2013, Carta dello spazio pubblico, http://www.biennalespaziopubblico.it/ wp-content/uploads/2016/12/CARTA_SPAZIO_PUBBLICO.pdf, (10/2021).

Lassini P. 2014, I sistemi verdi e la forestazione del fondovalle, in Bonardi L., Caligari A., Foppoli D., Gadola L., Grossi D., Stangoni T., Vanoi G. (a cura di), Paesaggi Valtellinesi. Trasformazione del territorio cultura e identità locale, Mimesis, Milano-Udine, pp. 159-170.

Lanzani A., Curci F. 2018, Le Italie in contrazione, tra crisi e opportunità, in De Rossi A. (a cura di), Riabitare l'Italia. Le aree interne tra abbandoni e riconquiste, Donzelli, Roma, pp. 79-107.

Lefebvre H. 1974, La production de l'espace, Editions Anthropos, Paris.

Maier L., Shobayashi M. 2001, Multifunctionality: Towards an Analytical Framework, OECD Publications Service, Paris.

Mattiucci C., De Marco R. 2013, Le nuove forme del territorio en débat. II paesaggio come strumento analitico e progettuale della post-metropoli, in AA.VV., Atti della XVI Conferenza Nazionale SIU. Urbanistica per una diversa crescita, «Planum. The Journal of Urbanism», n.27, vol.II.
Mattiucci C. 2018a, Il paesaggio come spazio pubblico. Interpretazioni da una ricerca sulle metropoli alpine, LISt Lab, Barcellona.

Mattiucci C. 2018b, Landscape as a Founding Element of the Contemporary Urban, «Built Environment», vol. 44, n. 3, pp. 315-325.

Mocarelli L. 2013, Spazi e diritti collettivi nelle aree montane: qualche riflessione su Alpie Appennini in età moderna, «Proposte e Ricerche», n. 70, pp. 173-202.

Pallottino G. 2014, Proprietà collettive e usi civici, «Scienze Del Territorio», vol. 1, pp. 433-438.

Perlik M. 2019, The Spatial and Economic Transformation of Mountain Regions, Routledge, Oxon and New York.

Pinelli C. A. 2020, La carta di Fontecchio e la protezione della natura e del paesaggio, in Casanova L. (a cura di), Avere cura della montagna. L'Italia di salva dalla cima, Altraeconomia, Milano, pp. 37-52.

Restelli S. 2021, II paesaggio alpino tra fragilità e resilienza, in Brunetta G., Caldarice O., Russo M., Sargolini M. (a cura di), Atti della XXIII Conferenza Nazionale SIU. DOWNSCALING, RIGHTSIZING. Contrazione demografica e Riorganizzazione spaziale, Politecnico di Torino, Planum Publisher, Roma-Milano, pp. 141-146.

Salsa A. 2019, I paesaggi delle Alpi, Donzelli, Roma.

Schama S. 1997, Paesaggio e memoria, Mondadori, Milano.

UN, United Nations 2015, Sustainable Development Coals. Services Section, Department of Public Information, United Nations, https://sdgs.un.org/goals/goal11 (05/2021).

Weil S., 2002, The Need for Roots, Routledge, London and New York. 